\title{
Ouverture Commerciale, Croissance Economique et Environnement au Cameroun : Une Etude Empirique de la Courbe Environnementale de Kuznets
}

\section{Nkwenka Nyanda Patrick Geoffroy, Ngassa Nya Yve Daniel,}

Docteurs en Sciences Economiques, Département d'Analyse et Politique Economiques, Faculté des Sciences Economiques et de Gestion, Laboratoire de Recherche en Economie et Management (LAREMA),

Université de Dschang, Cameroun

\section{Kaffo Fotio Hervé,}

Docteur en Sciences Economiques, Département d'Economie Publique et des Ressources Humaines, Faculté des Sciences Economiques et de Gestion,

Laboratoire de Recherche en Economie et Management (LAREMA),

Université de Dschang, Cameroun

Doi:10.19044/esj.2019.v15n25p387 URL:http://dx.doi.org/10.19044/esj.2019.v15n25p387

\section{Résumé}

L'objet de ce papier est de déterminer les effets de la croissance économique et de l'ouverture commerciale sur la qualité de l'environnement. La méthode de cointégration par les retards échelonnés ou Auto Regressive Distributed Lags (ARDL) développée par Pesaran et al. (2001) a été appliquée sur les données macroéconomiques du Cameroun sur la période allant de 1971 à 2011. Les résultats révèlent que : la croissance économique induit la détérioration de l'environnement à court terme, mais l'améliore à long terme tandis que l'ouverture commerciale détériore l'environnement au Cameroun.

Mots clés: Environnement, Croissance économique, Ouverture commerciale, Cameroun 


\title{
Trade Openness, Economic Growth and Environment in Cameroon: An Empirical Study of the Environmental Kuznets Curve
}

\section{Nkwenka Nyanda Patrick Geoffroy, Ngassa Nya Yve Daniel,}

Docteurs en Sciences Economiques, Département d'Analyse et Politique Economiques, Faculté des Sciences Economiques et de Gestion, Laboratoire de Recherche en Economie et Management (LAREMA),

Université de Dschang, Cameroun

\section{Kaffo Fotio Hervé,}

Docteur en Sciences Economiques, Département d'Economie Publique et des Ressources Humaines, Faculté des Sciences Economiques et de Gestion,

Laboratoire de Recherche en Economie et Management (LAREMA),

Université de Dschang, Cameroun

\begin{abstract}
The objective of this paper is to examine the effects of economic growth and the trade openness on the quality of the environment. Pesaran's et al (2001) Auto-Regressive Distributed Lags (ARDL) was applied on the macroeconomic data of Cameroon dating from 1971 to 2011. The results reveal that: the economic growth induces a deterioration of the environment in the short-term but improves it in the long-term while the trade openness deteriorates the environment in Cameroon.
\end{abstract}

Keywords: Environment, Economic Growth, Trade openness, Cameroon

\section{Introduction}

L'attention accordée aux problèmes environnementaux (réchauffement climatique) est de plus en plus préoccupante et occupe un espace médiatique très important. En effet, le réchauffement climatique, dû à l'accumulation des Gaz à Effet de Serre (GES), constitue une grande menace pour l'humanité (Stern, 2007).

Selon le Ministère de l'Environnement et de la Faune (MINEF, 2005), les émissions de dioxyde de carbone $\left(\mathrm{CO}_{2}\right)$ constituent le principal GES émis au Cameroun. En effet, les émissions de $\mathrm{CO}_{2}$ représentent 53,73\% des émissions totales de GES, suivies par les émissions de méthane $\left(\mathrm{CH}_{4}\right)$ 
avec $24,36 \%$, de protoxyde d'azote $\left(\mathrm{N}_{2} \mathrm{O}\right)$ avec $18,05 \%$ et de l'oxyde de carbone $(\mathrm{CO})$ qui pollue à hauteur de $3,3 \%$. Les autres GES sont responsables de moins de $1 \%$ des émissions. La figure 1 montre l'évolution des émissions totales de $\mathrm{CO}_{2}$ au Cameroun sur la période allant de 1965 à 2009.

Figure 1 : Evolution des émissions de $\mathrm{CO}_{2}$ au Cameroun de 1965 à 2009 (en kilotonnes)

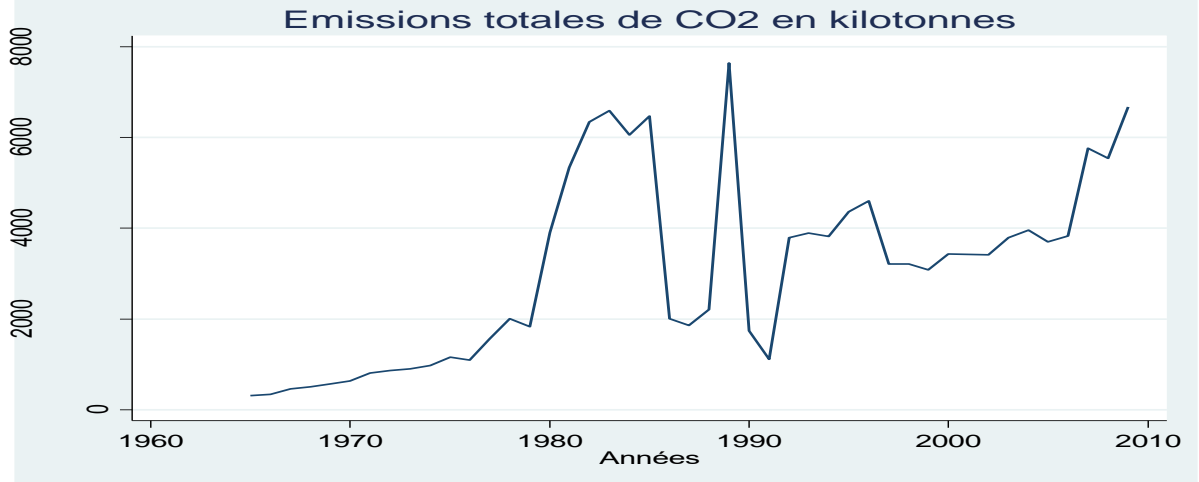

Source : Africa Development Indicators (2012)

La figure 1 révèle que les émissions de $\mathrm{CO}_{2}$ sont croissantes jusqu'en 1985, avec un pic de 6589 kilotonnes métriques en 1983, avant de connaitre une diminution brusque sur la période 1986/1988, ce qui semble coïncider avec l'entrée du Cameroun en récession. En dépit de la crise que traverse l'appareil productif camerounais, les émissions de $\mathrm{CO}_{2}$ atteignent le niveau record de 7638 millions de tonnes métriques en 1989, avant de connaitre une chute quasi-permanente à partir de 1990 et ce jusqu'à la fin de la décennie (néanmoins, on peut remarquer une période de hausse à partir de 1992, mais elle est très brève). A partir de l'an 2000, on assiste à une hausse permanente des émissions de $\mathrm{CO}_{2}$, qui ont presque doublé entre 2000 et 2009, passant ainsi de 3432 à 6673 tonnes métriques.

Cette augmentation des émissions de $\mathrm{CO}_{2}$ a poussé les autorités camerounaise à s'engager dans de vastes réformes qui devraient permettre de réaliser une croissance durable écologique au regard de la croissance économique enregistrée dans le pays depuis quelques années.

L'économie camerounaise a suivi, depuis son accession à l'indépendance, une évolution en trois (03) phases : deux décennies de relative prospérité (1965-1985), une décennie de récession économique aigüe (1985-1995) et depuis 1995, une certaine reprise de la croissance économique. La figure 2 retrace l'évolution du taux de croissance du PIB au cours de la période 1965-2011: 
Figure 2 : Evolution du taux de croissance du PIB au Cameroun de 1965 à 2011

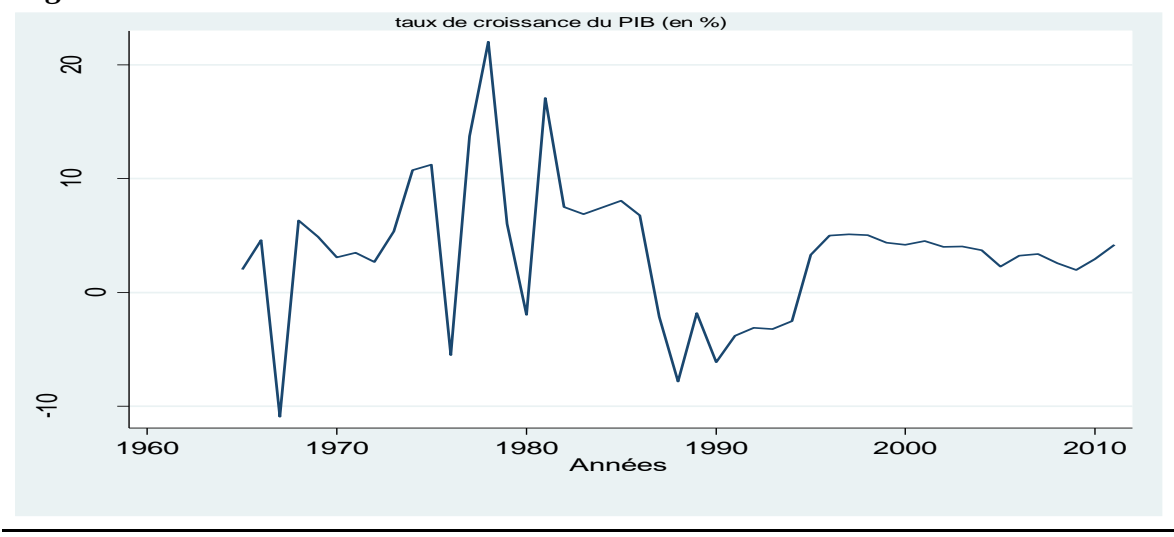

Source : Africa Development Indicators (2012)

En dépit de quelques années de croissance négative, on constate que l'économie camerounaise a connu une période de fort décollage entre 1965 et 1985, impulsée par une croissance spectaculaire du secteur pétrolier à partir de 1973 qui permet d'atteindre le taux de croissance record de $22 \%$ en 1978. A partir de 1986, le Cameroun entre en récession, avec des taux de croissance négatifs jusqu'en 1994. A la faveur de la dévaluation de 1994 et des programmes d'austérité mis en place, le Cameroun a retrouvé le sentier de la croissance à partir de 1995, même si le taux de croissance reste relativement faible.

Le Document de Stratégie pour la Croissance et l'Emploi (DSCE), élaboré en 2009, et les Objectifs de Développement Durable (fixés en 2016) réaffirment la volonté du gouvernement camerounais de poursuivre les objectifs de croissance économique, d'améliorer les conditions de vie des populations et de gérer durablement les ressources environnementales. Dès lors, on pourrait s'interroger, au regard des faits stylisés décrits ci-dessus, sur la relation qui existerait entre la croissance économique et l'environnement.

Cette relation a fait l'objet de nombreux débats dans la littérature, opposant «pessimistes » et « optimistes ». Pour les «pessimistes », à l'instar de Meadows et al. (1972), la dégradation de l'environnement est essentiellement due à l'activité économique (production et consommation) qui entraine l'épuisement des ressources naturelles, l'accumulation des déchets et la concentration des polluants qui détruisent les écosystèmes de manière irréversible. Les seconds par contre, notamment Kuznets (1955), estiment que la poursuite de la croissance économique va dans le sens de la protection de l'environnement à long terme. Ces derniers postulent une relation entre la croissance économique et les dommages environnementaux, selon laquelle l'environnement se détériore dans les pays pauvres et s'améliore ensuite au fur et à mesure que le pays s'enrichit (théorie de la Courbe Environnementale de Kuznets). 
Selon cette théorie, la solution aux dommages causés à l'environnement serait de favoriser le plus possible la croissance des activités de production et de consommation de biens et services, mesurées par le PIB réel par habitant (Banque mondiale, 1992). L'hypothèse est que, durant la première phase de développement économique d'un pays, il y a peu d'émissions polluantes car la production est faible, puis la pollution va s'accroitre car l'industrialisation impose une pression importante sur l'environnement. Enfin, à partir d'un certain niveau de revenu, les agents commencent à prendre conscience de la valeur d'un environnement sain et la conjugaison de la modification des préférences individuelles, de l'implication du pouvoir exécutif dans les problématiques environnementales et de l'augmentation relative de l'importance des services dans le PIB des économies les plus développées tend à diminuer les dégradations environnementales.

Depuis l'étude de Grossman et Krueger (1991) sur l'impact environnemental de l'Accord de Libre Echange Nord Américain, on distingue trois effets interdépendants: l'effet d'échelle, l'effet de composition et l'effet technique dans l'explication de la Courbe Environnementale de Kuznets (OMC, 1998).

L'effet d'échelle capture la relation entre l'expansion des activités économiques et l'évolution de la qualité de l'environnement et indique que pour un coefficient de pollution et une structure de l'économie donnés, tout accroissement de l'activité économique aura pour conséquence une augmentation de la pollution (OMC, 1998).

L'effet de composition quant à lui traduit la modification de la structure de production de l'économie induite par la spécialisation internationale des pays participants au commerce. L'impact (positif ou négatif) de l'effet de composition sur la qualité de l'environnement d'un pays dépendra de la nature des biens qu'il produit. En effet, si l'échelle de production et l'intensité de pollution sont constantes, alors les émissions polluantes dépendront de la structure de l'économie. Par conséquent, elles baisseront par l'effet de composition si l'économie se spécialise dans la production des biens propres, et augmenteront si le pays se spécialise dans des industries polluantes.

L'effet technique est le moyen par lequel la libéralisation du commerce influe sur le degré de pollution des industries et des ménages. Pour une échelle de production et une composition de l'économie données, les émissions polluantes globales dépendront des techniques de production, et dans la mesure où la mondialisation modifie ces techniques par le biais de l'innovation et des transferts technologiques, l'effet net sur la qualité de l'environnement sera positif. 
Il faut préciser que ces trois effets agissent différemment selon la position de la Courbe Environnementale de Kuznets (CEK). Dans la phase ascendante de la courbe, les effets d'échelle et de composition (due à la spécialisation dans les industries polluantes) dominent l'effet technique : en l'absence de technologies propres, l'expansion des activités économiques augmente les émissions polluantes. En revanche, lorsque les pays ont dépassé le point de retournement, l'effet de composition (due à la tertiarisation cette fois ci) et l'effet technique dominent l'effet d'échelle de telle sorte que la dégradation de l'environnement s'amoindrit dans les pays à haut revenu (Stern, 2003).

En résumé, l'ouverture commerciale favorise l'augmentation du revenu par habitant, l'innovation et la diffusion des technologies propres dans les économies en développement, ce qui réduit les émissions polluantes globales et participe à la préservation de la qualité de l'environnement. De plus, l'augmentation des revenus stimule la demande d'aménités environnementales (OCDE, 2011).

Cependant, force est de constater au regard des résultats des travaux empiriques que la théorie de la courbe environnementale de Kuznets ne se vérifie pas toujours (Phu Nguyen et Azomahou, 2002 ; Akpan et Chuku, 2011 ; Saboori et Soleymani, 2011).

L'objectif de ce papier est de déterminer les effets de la croissance économique et l'ouverture commerciale sur la qualité de l'environnement au Cameroun.

Le reste de l'article est organisé comme suit : la section 2 est consacrée à la démarche méthodologique, la section 3 est réservée à l'analyse des résultats et la section 4 présente la conclusion et quelques recommandations de politique économique et environnementale.

\section{Methodologie}

\subsection{Nature et source des données}

Les données utilisées dans ce papier sont quantitatives, annuelles et de source secondaire. Elles proviennent essentiellement de la base de données de la Banque Mondiale (Africa Development Indicators, 2012) et couvrent la période allant de 1971 à 2011.

\subsection{Description des variables}

Il s'agit de la variable expliquée (2.2.1) et des variables explicatives (2.2.2).

\subsubsection{Variable expliquée}

La variable expliquée est représentée par les émissions de CO2 par habitant. Approximée par l'indicateur $\mathrm{CO}$, il s'agit des émissions qui émanent de la combustion des combustibles fossiles et de la production de 
ciment (Africa Development Indicators, 2012). Cette variable dépendante est influencée par des variables dites explicatives.

\subsubsection{Variables explicatives}

Elles sont réparties en variables d'intérêt (2.2.2.1) et de contrôle (2.2.2.2).

\subsubsection{Variables d'intérêt}

Il s'agit essentiellement du PIB par habitant, du PIB par habitant élevé au carré et du degré d'ouverture commerciale. Ces variables sont construites sur la base de la plupart des travaux empiriques sur la CEK. Il s'agit notamment des travaux de Cissokho (2004), He (2007) et Saabori et Soleymani (2011).

\section{- PIB par habitant}

Le PIB par habitant, dont l'indicateur est $\mathrm{PIB}_{\mathrm{t}}$, représente la valeur du Produit Intérieur Brut divisée par la population totale en milieu d'année. Ce revenu correspond au revenu de la phase ascendante de la CEK. Etant donné que le Cameroun est un pays en développement, on peut espérer que le faible niveau de revenu par habitant amène les individus à privilégier leur bien être individuel par rapport à la qualité de l'environnement.

\section{* PIB par habitant au carré}

Le PIB par habitant au carré, avec pour indicateur $\mathrm{PIB}_{\mathrm{t}}{ }^{2}$, mesure le PIB moyen par habitant à long terme. Ce revenu correspond au revenu de la phase ascendante de la CEK. D'un point de vue théorique, le transfert de technologies propres des pays développés vers les pays en développement, l'existence d'une capacité à payer pour préserver l'environnement, de même que l'existence de normes environnementales strictes auront pour conséquence de diminuer l'intensité de pollution par unité de bien produit.

\section{Degré d'ouverture commerciale}

L'ouverture commerciale, mesurée par $\mathrm{OUV}_{\mathrm{t}}$, est le ratio de la somme des importations et des exportations par rapport au PIB. Le faible niveau de revenu par habitant couplé à l'absence d'une réglementation environnementale forte, font $\mathrm{du}$ Cameroun un potentiel « havre de pollution », ce qui risque d'accroitre les activités polluantes.

\subsubsection{Variables de contrôle}

Ces variables représentent l'effet de la structure de l'économie sur la dégradation de l'environnement. Elles renseignent particulièrement sur la contribution de l'industrie, de la densité de la population et de la consommation de carburant dans l'explication des émissions de $\mathrm{CO}_{2}$ au Cameroun.

\section{PIB industriel}

Le PIB industriel est la valeur ajoutée du secteur secondaire divisée par le PIB au terme d'une année. Approximé par l'indicateur IND $_{t}$, cette variable est introduite pour prendre en compte l'impact des activités industrielles sur la qualité de l'environnement au Cameroun. Compte tenu de 
la nature des industries et de l'absence d'une réglementation environnementale stricte en matière de pollution industrielle au Cameroun, on s'attend à un signe positif.

\section{* Densité de la population}

La densité de la population, appréhendé part $\mathrm{DPOP}_{\mathrm{t}}$, mesure le nombre d'habitants au kilomètre carré. Selon Malthus (1798), une population croissante présente des besoins alimentaires importants, ce qui créé des pressions sur les ressources environnementales.

\section{Consommation de carburant fossile (\%du total)}

La consommation de carburant fossile, dont l'indicateur est $\mathrm{CCARB}_{\mathrm{t}}$ renvoie à l'utilisation du charbon, du pétrole, l'huile de roche et des gaz naturels comme source d'énergie. L'utilisation d'énergie fossile est une source importante d'émissions de $\mathrm{CO}_{2}$. Au Cameroun, les émissions de $\mathrm{CO}_{2}$ dues à l'utilisation de l'énergie fossile proviennent essentiellement des transports, du résidentiel et des travaux de construction (MINEF, 2005).

L'ensemble des variables présentées ci-dessus peut être regroupé dans le tableau suivant :

Tableau 1 : Présentation des variables du modèle

\begin{tabular}{|l|c|l|}
\hline Variable retenue & Abréviation & \multicolumn{1}{|c|}{ Mesure } \\
\hline $\mathrm{CO}_{2}$ par habitant & $\mathrm{CO}$ & $\begin{array}{l}\text { Emissions totales de } \mathrm{CO}_{2} \text { (en tonnes métriques) } \\
\text { divisées par la population totale }\end{array}$ \\
\hline PIB par habitant & PIB & PIB moyen à court terme \\
\hline $\begin{array}{l}\text { PIB par habitant au } \\
\text { carré }\end{array}$ & PIB $^{2}$ & PIB moyen à long terme \\
\hline PIB industriel & IND & Valeur ajoutée industrielle divisée par le PIB \\
\hline $\begin{array}{l}\text { Degré d'ouverture } \\
\text { commerciale }\end{array}$ & OUV & $\begin{array}{l}\text { Ratio de la somme des importations et des } \\
\text { exportations par rapport au PIB }\end{array}$ \\
\hline $\begin{array}{l}\text { Densité de la } \\
\text { population }\end{array}$ & DPOP & Nombre d'habitants au kilomètre carré \\
\hline $\begin{array}{l}\text { Consommation de } \\
\text { carburant fossile }\end{array}$ & CCARB & Consommation de carburant fossile (\% du total) \\
\hline
\end{tabular}

Source : Auteur, d'après la littérature

(Grossman et Krueger, 1991 ; He, 2005 ; Sharma, 2010)

Par ailleurs, les signes attendus des variables présentées ci-dessus sont regroupés dans le tableau suivant :

Tableau 2 : Récapitulatif des signes attendus

\begin{tabular}{|c|c|c|c|c|c|c|}
\hline Variables explicatives & $\mathrm{PIB}_{\mathrm{t}}$ & $\mathrm{PIB}_{\mathrm{t}}{ }^{2}$ & $\mathrm{IND}_{\mathrm{t}}$ & $\mathrm{OUV}_{\mathrm{t}}$ & $\mathrm{DPOP}_{\mathrm{t}}$ & $\mathrm{CCARB}_{\mathrm{t}}$ \\
\hline Signes attendus & + & - & + & + & + & + \\
\hline
\end{tabular}

Source: Auteur, d'après la littérature

(Grossman et Krueger, 1991 ; He, 2005 ; Sharma, 2010) 


\subsection{Modèle économétrique}

A partir de la littérature et en fonctions des données disponibles, nous avons choisi les émissions de $\mathrm{CO}_{2}$ par habitant comme indicateur de la qualité de l'environnement. Le modèle économétrique adopté s'inspire d'une littérature riche et diversifiée. Ainsi, à partir des travaux de Cissokho (2004), Halicioglu (2008), Sharma (2010), Akpan et Chuku (2011), le modèle économétrique suivant est retenu :

$\mathrm{CO}_{\mathrm{t}}=\alpha_{0}+\alpha_{1} \mathrm{PIB}_{\mathrm{t}}+\alpha_{2} \mathrm{PIB}_{\mathrm{t}}^{2}+\alpha_{3} \mathrm{IND}_{\mathrm{t}}+\alpha_{4} \mathrm{OUV}_{\mathrm{t}}+\alpha_{5} \mathrm{DPOP}_{\mathrm{t}}+\alpha_{6} \mathrm{CCARB}_{\mathrm{t}}+$

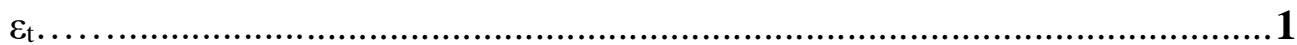

Avec: $\mathrm{CO}_{\mathrm{t}}$ les émissions de $\mathrm{CO}_{2}$ en tonnes métriques par habitant, $\mathrm{PIB}_{t}$ et $\mathrm{PIB}_{t}{ }^{2}$ restent inchangés, IND ${ }_{t}$ le PIB industriel, $\mathrm{OUV}_{t}$ le taux d'ouverture commerciale, DPOP ${ }_{t}$ est la densité de la population, CCARB $_{t}$ est la consommation de carburant (en \% du total), $\varepsilon_{t}$ le terme d'erreur et les $\alpha_{t}$ sont les paramètres à estimer.

\subsection{Méthode d'estimation}

L'approche de cointégration par les modèles autorégressifs à retards échelonnés (Auto Regressive Distributed Lags ou ARDL) développée par Pesaran et al. (2001) est privilégiée.

Le choix de cette méthode se justifie par les avantages suivants :

- D'abord, l'ARDL, contrairement aux tests de cointégration classiques n'exige pas que toutes les variables du modèle soient intégrées au même ordre $^{1}$. L'ARDL est plus flexible et présente donc l'avantage d'être applicable lorsque toutes les variables sont $\mathrm{I}(0)$, I(1), ou mutuellement intégrées. Toutefois, la méthode cesse d'être applicable lorsque l'ordre d'intégration des variables est supérieure à 1 ;

- Ensuite, l'ARDL offre un éventail de choix relatifs au nombre de variables endogènes et exogènes et au retard optimal à introduire dans le modèle. De plus, cette flexibilité de l'ARDL permet d'introduire les variables dummy dans le test de cointégration, contrairement à la technique de Johansen qui n'autorise pas une telle inclusion.

Traditionnellement, Pesaran et al. (2001) proposent d'estimer le modèle à correction d'erreur sans restriction (ou Unrestricted Error Correction Model) suivant $^{2}$ :

$\Delta \mathrm{Q}_{\mathrm{t}}=\beta_{0}+\pi_{\mathrm{QQ}} \mathrm{Q}_{\mathrm{t}-1}+\pi_{\mathrm{QX}} \mathrm{X}_{\mathrm{t}-1}+\sum_{\mathrm{i}=1}^{\mathrm{p}} \delta_{\mathrm{i}} \Delta \mathrm{Q}_{\mathrm{t}-\mathrm{i}}+\sum_{\mathrm{j}=0}^{\mathrm{q}} \vartheta_{\mathrm{j}} \mathrm{X}_{\mathrm{t}-\mathrm{j}}+\mu_{\mathrm{t}}$

Où : $\pi_{\mathrm{QQ}}$ et $\pi_{\mathrm{QX}}$ sont les coefficients de long terme, $\beta_{0}$ le terme constant, $\Delta$ et $\sum$ sont les opérateurs de différence première et de somme, $\mu_{\mathrm{t}}$ est le terme

${ }^{1}$ Rappelons qu'une série non stationnaire est dite intégrée d'ordre p i.e. I(p), si elle doit être différenciée $\mathrm{p}$ fois avant d'être stationnaire.

2 Cette spécification a été proposée par Akpan et Akpan (2012) dans l'article intitulé

«Electricity consumption, carbon Emissions and Economic Growth in Nigeria », p.6 
d'erreur, $\Delta \mathrm{Q}_{\mathrm{t}-\mathrm{j}}$ représente les valeurs décalées de la variable dépendante et $\Delta \mathrm{X}_{\mathrm{t}-\mathrm{i}}$ représente les variables présentes et décalées des variables explicatives.

\subsubsection{Description de la démarche d'estimation d'un modèle ARDL}

L'estimation d'un modèle ARDL se déroule en trois étapes : la première est relative au test de stationnarité (2.4.1.1), la seconde consiste à déterminer le retard optimal à introduire dans le modèle (2.4.1.2) et la troisième est réservée au test de cointégration (2.4.1.3).

\subsubsection{Test de stationnarité}

Dans cette étude, deux types de test de racine unitaire sont utilisés : le test de Dickey-Fuller Augmenté (DFA) et le test de Phillips-Perron (PP).

Le test de DFA est une extension du test de Dickey-Fuller Simple. Ce test consiste à tester la présence d'une racine unitaire dans le polynôme associé à la dynamique autorégressive du processus.

Les hypothèses nulles et alternatives à tester sont définies comme suit :

$$
\begin{aligned}
& \mathrm{H}_{0}: \boldsymbol{\rho}=0 \text {, i.e. le processus est non stationnaire } \\
& \mathrm{H}_{1}: \boldsymbol{\rho}<1 \text {, i.e. le processus est stationnaire }
\end{aligned}
$$

Si la statistique DFA est supérieure à la valeur critique du test pour un seuil de signification donné, alors on accepte l'hypothèse de la stationnarité de la série. Dans le cas contraire, on accepte l'hypothèse de la non stationnarité.

Le test de PP est construit sur correction non paramétrique des statistiques de Dickey-Fuller pour prendre les erreurs hétoroscédastiques. Les hypothèses du test sont les mêmes que celles du test DFA.

Le choix de ces deux techniques est dû au fait qu'elles sont les plus utilisés pour l'analyse des séries temporelles.

\subsubsection{Choix du retard optimal}

Après avoir vérifié que toutes les variables respectent les conditions d'application de l'ARDL (i.e. qu'elles sont au plus I (1)), il revient maintenant d'estimer le retard optimal que l'on doit introduire aux variables de l'équation 2. Toutefois, pour déterminer le retard optimal $\mathrm{p}^{*}$, il convient de fixer le retard maximum possible $\mathrm{P}_{\max }$, défini tel que $\mathrm{P}_{\max }=\left[4\left(\frac{N}{100}\right)^{\frac{2}{9}}\right]$ (Lélé, 2010), où $\mathrm{N}$ est la taille de l'échantillon.

Après avoir déterminé $\mathrm{P}_{\max }$, le choix du retard optimal est guidé par les critères d'information de Akaike (AIC) et de Schwarz (SIC) et sera égal au retard qui minimise les fonctions de Akaike et de Schwarz (Bourbonnais, 2007). La détermination du retard optimal $P^{*}$ conduit enfin à estimer tous les 
modèles ARDL $(q, p)^{3}$ dans lesquels les retards des séries varient entre 0 et $\mathrm{P}^{*}$, et l'ARDL optimal est celui qui minimise les critères d'information (AIC et SIC).

\subsubsection{Test de cointégration}

Le choix de l'ARDL optimal implique de tester l'existence d'une relation de long terme entre les variables de l'équation 2. Pesaran et al. (2001) proposent de tester les hypothèses suivantes:

$\mathrm{H}_{0}: \pi_{\mathrm{QQ}}=\pi_{\mathrm{QX}}=0$

$\mathrm{H}_{1}: \pi_{\mathrm{QQ}} \neq \pi_{\mathrm{QX}} \neq 0$, ou $\pi_{\mathrm{QQ}} \neq 0$ et $\pi_{\mathrm{QX}}=0$, ou $\pi_{\mathrm{QQ}}=0$ et $\pi_{\mathrm{QX}} \neq 0$

La statistique du test est la F-statistique ou la Wald statistique. Celleci ne suit pas une loi standard du type loi normale, loi de Student ou loi de Fisher (Lélé, 2010). Pour pallier à ce problème, Pesaran et al. (2001) proposent deux ensembles de valeurs critiques auxquelles est comparée la Fstatistique : le premier ensemble correspond au cas où les variables sont $\mathrm{I}(0)$ et représentent la borne inférieure et le second, celui où toutes les variables sont I(1) et représente la borne supérieure ${ }^{4}$. Après comparaison, respecter l'une des règles de décision suivante : si F-statistique inférieure à la borne inférieure, alors il n' y a pas de relation de cointégration; si F-statistique comprise entre la borne inférieure et la borne supérieure, le test n'est pas concluant et on ne peut tirer de conclusion et si la F-statistique est supérieure à la borne supérieure, alors, l'hypothèse de cointégration est acceptée.

Dans la mesure où l'hypothèse de cointégration est validée, l'étape suivante consiste à spécifier une équation de long terme et le Mécanisme à Correction d'Erreur (MCE) en vue de prendre en compte les éventuels déséquilibres qui peuvent exister à court terme. Le coefficient du terme de correction d'erreur (ECT(-1)) doit être négatif et significatif afin de confirmer l'existence d'une relation de cointégration.

\subsubsection{Tests de validation}

\subsubsection{Test de détection de l'autocorrélation}

Le test de Breusch-Godfrey est utilisé dans ce papier. Ce choix est dû au fait qu'il permet de tester l'autocorrélation d'un ordre supérieur. Le test porte essentiellement sur le terme d'erreur ou résidu $\left(\boldsymbol{\varepsilon}_{\mathbf{t}}\right)$ et les hypothèses à tester sont :

\footnotetext{
${ }^{3}$ Dans cette expression, $\mathrm{q}$ et $\mathrm{p}$ représentent le nombre de retards imposé à chaque variable et ce nombre est compris entre 0 qui le retard minimal et $\mathrm{P}^{*}$ qui est le retard optimal à prendre en compte lors de l'estimation de la relation de court terme entre les variables

${ }^{4}$ Raison pour laquelle cette procédure est encore appelée «bounds testing approach » ou « approche de test par intervalle ».
} 
- $\mathrm{H}_{0}: \operatorname{cov}\left(\varepsilon_{\mathrm{t}} ; \varepsilon_{\mathrm{t}-\mathrm{p}}\right)=0$, i.e. pas d'autocorrélation ;

$-\mathrm{H}_{1}: \operatorname{cov}\left(\varepsilon_{\mathrm{t}} ; \varepsilon_{\mathrm{t}-\mathrm{p}}\right) \neq 0$, i.e. il y’a autocorrélation.

La statistique LM qui est distribuée comme un Khi-deux $\left(\chi^{2}\right)$ à $p$ degré de liberté ou retards est utilisée. Par conséquent, si $\mathrm{n} * \mathrm{R}^{2}>\chi^{2}(\mathrm{p})$ calculé au seuil de signification $\alpha$, alors l'hypothèse d'absence d'autocorrélation est rejetée, sinon elle est acceptée. $\mathrm{R}^{2}$ est le coefficient de détermination.

\subsubsection{Test de détection de l'hétéroscédasticité}

Le test de White permet de la détecter. L'idée de base consiste à vérifier si les carrés des résidus sont expliqués par les variables du modèle, auquel cas il y a hétéroscédasticité. Ce test utilise la statistique du Khi-deux $\left(\chi^{2}\right)$ en vue de tester les hypothèses. Pour un processus $\boldsymbol{\varepsilon}_{\mathbf{t}}$ donné, les hypothèses suivantes seront testées :

- $\mathrm{H}_{0}: \operatorname{var}\left(\varepsilon_{t}\right)=\boldsymbol{\sigma}^{2}$, i.e. le modèle est homoscédastique ;

$-\mathrm{H}_{1}: \operatorname{var}\left(\varepsilon_{\mathrm{t}}\right)=\boldsymbol{\sigma}_{\mathrm{t}}^{2}$, i.e. le modèle est hétéroscédastique.

La règle de décision consiste à comparer les valeurs critiques $\left(\chi^{2} l u\right)$ aux valeurs obtenues $\left(\chi^{2} \mathrm{cal}\right)$ de la statistique $\mathrm{F}$. Si au seuil de signification $(\alpha), \chi^{2} c a l>\chi^{2} l u$, on rejette l'hypothèse nulle $\left(\mathrm{H}_{0}\right)$; ou encore si $\mathrm{P}$-value $<\alpha$, on rejette $\mathrm{H}_{0}$ et dans le cas contraire, rejeter $\mathrm{H}_{1}$.

Si les résidus sont homoscédastiques et non autocorrélés, la méthode des Moindres Carrés Ordinaires (MCO) est recommandé pour estimer les paramètres du modèle.

\subsubsection{Test de stabilité des coefficients}

Les tests Cumulative Sum (CUSUM) et Cumulative Sum of Square (CUSUMQ) permettent de tester la stabilité des coefficients estimés. Le test CUSUM teste les instabilités structurelles, tandis que le test CUSUMQ teste les instabilités ponctuelles ou conjoncturelles. Les coefficients sont déclarés stables au seuil $\alpha$ si les statistiques des tests CUSUM et CUSUMQ évoluent strictement à l'intérieur du seuil critique (stylisé par les traits en rouge). Dans le cas contraire, rejeter l'hypothèse de la stabilité des coefficients.

\subsubsection{Tests de significativité}

\subsubsection{Test de Student}

Il est applicable lorsque la taille de l'échantillon est inférieure à 30 . $\mathrm{Au}$ cas contraire, la valeur lue dans la table sera celle correspondant à la valeur infinie ou celle de la table de la loi normale (Bourbonnais, 2002). Il se déroule ainsi : après avoir défini un seuil de significativité $\alpha$, déterminer le degré de liberté qui est égal à n-k-1 (n est la taille de l'échantillon, $\mathrm{k}$ est le nombre de variables exogènes) et poser les hypothèses à tester.

$$
\begin{aligned}
& \text { > } \mathrm{H} 0: \beta i=0 \\
& \text { } 1: \beta i \neq 0
\end{aligned}
$$


Pour la valeur estimée d'un paramètre, la valeur calculée de la statistique s'obtient à partir de la formule suivante :

tcal $=($ valeur estimée - valeur réelle)/écart-type de la valeur estimée

Le calcul de cette valeur permet de la comparer à la valeur lue dans la table qui est : $t l u=t_{\alpha / 2(n-k-1)}$ pour une valeur de $\alpha$ choisie. Si $t_{c a l}<t_{l u e}$, alors, on accepte l'hypothèse nulle et on conclut que le paramètre n'est pas significatif.

\subsubsection{Test de Fisher}

Il s'effectue sur la base de la valeur du coefficient de détermination $\mathrm{R}^{2}$ (Chauvat, 2003). Comme le test précédent, on choisit d'abord un seuil de signification, puis, on cherche la valeur calculée de la statistique sur la base de la formule $: F_{c a l}=\frac{(n-k) R^{2}}{(k-1)\left(1-R^{2}\right)}$. Cette valeur est comparée à celle lue dans la table de Fischer à (k-1, n-k-1) degré de liberté.

Les hypothèses sur lesquelles repose ce test sont les suivantes :

$>\mathrm{H} 0: \beta 1=\beta 2=\beta 3=\beta 4=\beta 5=0$

$>\mathrm{H} 1: \beta 1 \neq \beta 2 \neq \beta 3 \neq \beta 4 \neq \beta 5 \neq 0$

La règle de décision est la suivante: si $F_{c a l}<F_{\text {lue }}$, on accepte l'hypothèse nulle, et le modèle n'est pas globalement significatif. Dans le cas contraire, accepter l'hypothèse alternative.

\section{Resultats}

\subsection{Tests préliminaires}

\subsubsection{Test de stationnarité}

Les résultats du tableau 3 indiquent des ordres d'intégrations identiques pour les tests DFA et PP. En effet, les variables CO et DPOP sont $\mathrm{I}(0)$, tandis que les variables PIB, $\mathrm{PIB}^{2}$, IND, OUV et CCARB sont I(1), rendant ainsi l'application du test de cointégration de Pesaran et al. (2001) inévitable. Toutefois, il convient d'abord de déterminer le retard optimal.

Tableau 3 : Résultats du test de stationnarité

\begin{tabular}{|c|c|c|c|c|c|}
\hline \multirow{2}{*}{ Variable } & \multicolumn{2}{|c|}{ Test de Dickey-Fuller Augmenté } & \multicolumn{2}{c|}{ Test De Philips-Perron } & \multirow{2}{*}{ Décision } \\
\cline { 2 - 5 } & Niveau & $\begin{array}{c}\text { Différence } \\
\text { première }\end{array}$ & Niveau & $\begin{array}{c}\text { Différence } \\
\text { première }\end{array}$ & \\
\hline CO & $-3,115729 * *$ & - & $-3,069718^{* *}$ & - & $\mathrm{I}(0)$ \\
\hline PIB & 0,583424 & $-3,791316^{*}$ & $-1,992088$ & $-3,765766^{*}$ & $\mathrm{I}(1)$ \\
\hline PIB $^{2}$ & 0,168339 & $-3,601663^{*}$ & $-0,220618$ & $-3,573490^{*}$ & $\mathrm{I}(1)$ \\
\hline
\end{tabular}




\begin{tabular}{|c|c|c|c|c|c|}
\hline IND & 0,537137 & $-5,164890^{*}$ & 0,460049 & $-5,164890^{*}$ & $\mathrm{I}(1)$ \\
\hline OUV & 0,057745 & $-5,860397^{*}$ & 0,242733 & $-5,856715^{*}$ & $\mathrm{I}(1)$ \\
\hline DPOP & $-5,578742 *$ & - & $9,063280 *$ & & $\mathrm{I}(0)$ \\
\hline CCARB & 1,634432 & $-6,437472 *$ & 1,736062 & $-6,435487 *$ & $\mathrm{I}(1)$ \\
\hline
\end{tabular}

Note : ${ }^{*}{ }^{* *}$ dénotent respectivement $1 \%$ et $5 \%$ et représentent le degré de significativité des variables. Une autre solution consiste à comparer les statistiques de test aux valeurs critiques de MacKinnon (1996) proposées par le logiciel.

Source : Auteur, à partir du logiciel Eviews 5.0

\subsubsection{Détermination du retard optimal}

Le retard maximal à retenir pour le modèle est $3\left(\mathrm{p}_{\max }=3\right)$ et le retard optimal qui assure la minimisation des critères d'information de Schwarz et de Akaike est également 3 (calcul effectué dans le logiciel R). Ce résultat est conforme à celui calculé dans Eviews et présenté à l'annexe 1. De plus, les investigations révèlent que le modèle autorégressif optimal, au vue des critères d'information, est ARDL $(2,0,1,2,2,2,1)^{5}$.

\subsubsection{Résultats du test de cointégration}

Le test de cointégration est effectué sur le modèle $\operatorname{ARDL}(2,0,1,2$, $2,2,1)$ optimal et les résultats du test sont présentés dans le tableau 4. La statistique du test F-stat $=7.96492$ étant supérieure à la borne supérieure lue dans la table CI(iii) Case III de Pesaran et al. (2001), il existe une relation de long terme entre les variables.

Tableau 4 : Résultats du test de cointégration

\begin{tabular}{|c|c|c|c|c|}
\hline F-Statistique & $\begin{array}{c}\text { Valeurs } \\
\text { critiques }\end{array}$ & $\begin{array}{c}\text { Borne } \\
\text { inférieure }\end{array}$ & $\begin{array}{c}\text { Borne } \\
\text { supérieure }\end{array}$ & Décision \\
\hline \multirow{3}{*}{$7,96492^{*}$} & $1 \%$ & 2,96 & 4,26 & Au seuil de $1 \%$, il \\
& $5 \%$ & 2,32 & 3,50 & existe une relation \\
& $10 \%$ & 2,03 & 3,13 & de long terme \\
entre les variables.
\end{tabular}

Note : Les valeurs critiques ont été extraites de la table proposée par Pesaran et al. (2001, p.300), table CI(iii), Case III : Unrestricted intercept and no trend, k=7. *est le degré de significativité à $1 \%$

Source : Auteur, calculé à partir du logiciel $R$

Etant donné l'existence d'une relation de long terme entre les variables, la prochaine étape consiste à estimer les dynamiques de long terme (représentée par l'équation 3 et le MCE décris à l'annexe 2). Mais avant, la validité du modèle retenu doit être testée.

${ }^{5}$ Les valeurs entre parenthèses indiquent le nombre de retards à inclure dans le MCE pour chaque variable. 
$\Delta y_{t}=\rho y_{t}-1+\sum_{k=1}^{p} \gamma_{k} \Delta y_{t-k}+\varepsilon_{t}$

\subsection{Tests de validation}

Cette section a pour but de présenter les résultats des tests de validation du modèle à estimer. Il s'agit de présenter tour à tour les résultats du test de l'autocorrélation, du test de l'hétéroscédasticité et du test de stabilité.

\subsubsection{Test de l'autocorrélation}

Les résultats du test présentés dans le tableau 5 montrent que le modèle retenu ne souffre pas de problème d'autocorrélation des erreurs car la statistique du test est inférieure à la valeur critique fournie par la table du khi deux. En effet, $N^{*} R^{2}=3,195699$ est inférieure au $\chi_{(30,01)}^{2}=50.89$ lu dans la table du khi deux. Ce résultat est confirmé par la p-value $(0,202331)$ qui est supérieure au seuil de 5\%. Par conséquent, l'hypothèse nulle d'absence d'autocorrélation est acceptée.

Tableau 5 : Résultats du test de l'autocorrélation

\begin{tabular}{|l|l|l|l|}
\hline \multicolumn{4}{|c|}{ Test de l'autocorrelation de Breusch-Godfrey: } \\
\hline F-statistique & 1.345857 & P-value & 0.275105 \\
\hline $\mathrm{N} * \mathrm{R}^{2}$ & 3.195699 & P-value & 0.202331 \\
\hline
\end{tabular}

\subsubsection{Test de l'hétéroscédasticité}

D'après le tableau 6, les résidus sont homoscédastiques (i.e. qu'ils ont une variance constante) car la statistique du test est inférieure à la valeur critique fournie par la table du khi deux. En effet, $\mathrm{N}^{*} \mathrm{R}^{2}=12,94625$ est inférieure au $\chi_{(30,01)}^{2}=50.89$ lu dans la table du khi deux. Ce résultat est confirmé par la $p$-value $(0,296853)$ qui est supérieure au seuil de $1 \%$.

Tableau 6 : Résultats du test de détection de l'hétéroscédasticité

\begin{tabular}{|l|l|l|l|}
\hline \multicolumn{4}{|c|}{ Test de détection de l'hétéroscédasticité de White: } \\
\hline F-statistique & 1.218097 & P-value & 0.320755 \\
\hline $\mathrm{N}^{*} \mathrm{R}^{2}$ & 12.94625 & P-value & 0.296853 \\
\hline
\end{tabular}

Source : Auteur, à partir d'Eviews 5.0

\subsubsection{Test de stabilité des coefficients}

Les tests CUSUM et CUSUMQ ont permis d'apprécier la stabilité des coefficients. Il ressort de ces tests que les paramètres estimés sont stables à long et à court termes au seuil de 5\% (voir annexes 3 et 4). 
Tableau 7 : Synthèse des résultats des tests préliminaires

\begin{tabular}{|c|c|c|}
\hline Test & Observations & Conclusion \\
\hline $\begin{array}{c}\text { Test de détection de } \\
\text { l'autocorélation }\end{array}$ & $\begin{array}{c}\chi^{2}=3,195699 \\
\text { P-value }=0,202331>1 \%\end{array}$ & $\begin{array}{c}\text { Les erreurs sont non } \\
\text { autocorrelés à 1\% }\end{array}$ \\
\hline $\begin{array}{c}\text { Test de détection de } \\
\text { l'hétéroscédasticité }\end{array}$ & $\begin{array}{c}\chi^{2}=12,9462 \\
\text { P-value }=0,296853>1 \%\end{array}$ & $\begin{array}{c}\text { Les erreurs sont } \\
\text { homoscédastiques à 1\% }\end{array}$ \\
\hline $\begin{array}{c}\text { Tests de stabilité des } \\
\text { paramètres }\end{array}$ & $\begin{array}{c}\text { Toutes les lignes bleues évoluent } \\
\text { à l'intérieur du corridor }\end{array}$ & $\begin{array}{c}\text { Les paramètres sont tous } \\
\text { stables à 5\% }\end{array}$ \\
\hline
\end{tabular}

Source : Auteurs, à partir d'Eviews 5.0

Les hypothèses de non autocorrélation et d'homoscédasticité des erreurs donnent lieu à l'estimation de l'équation 1 par la méthode des MCO.

\subsection{Résultats des estimations et interprétation}

L'hypothèse de la cointégration ayant été validée, il est reste à estimer la relation de long terme (3.3.1) et la relation de court terme (3.3.2) par la méthode des MCO.

\subsubsection{Relation de long terme}

Les coefficients de long terme ont été estimés par la méthode des MCO et les résultats sont présentés dans le tableau 8.

Tableau 8 : Résultats de l'estimation du modèle de long terme

\begin{tabular}{|c|l|l|l|l|}
\hline \multicolumn{5}{|c|}{ Variable dépendante : CO } \\
\hline $\begin{array}{c}\text { Variables } \\
\text { indépendantes }\end{array}$ & Coefficient & Ecart type & $\mathrm{t}$ (p-value) & $\begin{array}{l}\text { Degré de } \\
\text { significativité }\end{array}$ \\
\hline C & $-1,909904$ & 0,554367 & $\begin{array}{l}-3,445195 \\
(0,0016)\end{array}$ & $1 \%$ \\
\hline PIB & 0,005089 & 0,001754 & $\begin{array}{l}2,902154 \\
(0,0066)\end{array}$ & $1 \%$ \\
\hline PIB 2 & $-4,00 \mathrm{E}-06$ & $1,32 \mathrm{E}-06$ & $\begin{array}{l}-3,021611 \\
(0,0048)\end{array}$ & $1 \%$ \\
\hline IND & 0,024655 & 0,004830 & $\begin{array}{l}5,104734 \\
(0,0000)\end{array}$ & $1 \%$ \\
\hline OUV & 0,005530 & 0,001809 & $\begin{array}{l}3,057247 \\
(0,0044)\end{array}$ & $1 \%$ \\
\hline DPOP & $-0,021707$ & 0,004229 & $\begin{array}{l}-5,133074 \\
(0,0000)\end{array}$ & $1 \%$ \\
\hline
\end{tabular}




\begin{tabular}{|c|c|c|c|c|}
\hline CCARB & 0,013620 & 0,005159 & $\begin{array}{l}2,639909 \\
(0,0126)\end{array}$ & $5 \%$ \\
\hline \multicolumn{5}{|c|}{ Point de retournement $=636,125$} \\
\hline \multicolumn{2}{|c|}{$\mathrm{R}^{2}=0,700563$} & \multicolumn{3}{|c|}{ F-statistique $=12,86779$} \\
\hline \multicolumn{2}{|c|}{$\mathrm{R}^{2}$-ajusté $=0,646120$} & \multicolumn{3}{|c|}{ Prob. $($ F-statistique $)=0,0000$} \\
\hline
\end{tabular}

Source : Auteur, estimé à partir d'Eviews 5.0

Ces résultats font l'objet d'une analyse générale, puis spécifique dans les sections suivantes.

\subsubsection{Analyse générale du modèle}

Le coefficient de détermination est de 70,05\% (supérieur à 50\%), de même que le coefficient de détermination ajusté qui se fixe à $64,61 \%$. Ce résultat indique simplement que 64,61\% des émissions de $\mathrm{CO}_{2}$ par habitant au Cameroun sont expliquées par les variables explicatives du modèle, soit PIB, PIB $^{2}$, IND, OUV, DPOP et CCARB. De plus, la probabilité de la statistique, sensiblement égale à zéro, indique que le modèle est globalement significatif. En effet, Prob (F-statistique) $=0,0000<1 \%$, ce qui implique que notre modèle est globalement bien spécifié et peut donc servir à de prévisions économiques.

En outre, on relève que les variables $\mathrm{PIB}_{t}, \mathrm{PIB}_{\mathrm{t}}^{2}, \mathrm{IND}_{\mathrm{t}}, \mathrm{OUV}_{\mathrm{t}}, \mathrm{DPOP}_{\mathrm{t}}$ sont significatives au seuil de $1 \%$ et $\mathrm{CCARB}_{\mathrm{t}}$ au seuil de $5 \%$. Ces variables sont donc pertinentes dans l'explication de la qualité de l'environnement au Cameroun.

\subsubsection{Analyse spécifique des variables}

PIB par habitant $\left(\right.$ PIB $\left._{\mathbf{t}}\right)$

Le signe de cette variable est positif et conforme aux attentes. De plus, elle est significative et permet d'entrevoir que toute augmentation unitaire du PIB aura pour effet une augmentation des émissions de $\mathrm{CO}_{2}$ par tête de 0,005089 unités. Ce coefficient, bien que positif, est de loin inférieur à celui obtenu par Cissokho (2004) pour le Sénégal et Halicioglu (2008) pour la Turquie, qui obtiennent respectivement des élasticités égales à 59,79 et 12,31 .

\section{* PIB par habitant au carré ou de long terme $\left(\mathrm{PIB}_{\mathrm{t}}{ }^{2}\right)$}

Tout comme pour la variable $\mathrm{PIB}_{\mathrm{t}}$, le coefficient de $\mathrm{PIB}_{\mathrm{t}}{ }^{2}$ est significatif et conforme à la prévision théorique. Elle confirme la tendance à la baisse des dégradations environnementales à partir d'un certain niveau de revenu. En effet, elle indique que toute augmentation du PIB de long terme de $1 \%$ est associée à une réduction des émissions de $\mathrm{CO}_{2}$ de $4,00 \mathrm{E}-06 \%$, et même si la réduction est marginale, il reste qu'elle est conforme aux résultats de Cissokho (2004). 
De ces deux premières analyses, il ressort que l'hypothèse de la CEK entre le PIB par tête et les émissions de $\mathrm{CO}_{2}$ est vérifiée. Ce résultat est conforme à ceux de Holtz-Eakin et Selden (1995), Cissokho (2004) Halicioglu (2008) et Lean et Smyth (2009). Toutefois, le revenu optimal, qui se situe à $636,125 \$$ constants 2000 , est largement inférieur au seuil de 35428 \$ de Holtz-Eakin et Selden (1995) et au seuil de 9549\$ trouvé par Cissokho (2004).

\section{* Degré d'ouverture commerciale $\left(O U V_{t}\right)$}

Le degré d'ouverture commerciale a un impact positif et significatif sur les émissions de $\mathrm{CO}_{2}$ par habitant au Cameroun. Ainsi, toute augmentation du degré d'ouverture de $1 \%$ se solde par la hausse des émissions polluantes de $0,00553 \%$. Bien que très faible, le signe de cette variable est conforme à la plupart des travaux empiriques, notamment ceux de Halicioglu (2008), Sharma (2010) et Akpan et Chuku (2011).

D’une part, les exportations camerounaises sont dominées par les exportations d'hydrocarbures et de bois (respectivement 50,1\% et 12,5\% des exportations totales au premier semestre 2011) (INS, 2011), activités de plus en plus décriées comme polluantes. Par exemple, l'exploitation forestière, qui représente près de $13 \%$ des recettes de l'Etat, est considérée comme une des causes principales des émissions de $\mathrm{CO}_{2}$, et serait même responsable de $16 \%$ des émissions mondiales de $\mathrm{CO}_{2}$.

D’autre part, le Cameroun peut être considéré comme un « havre de pollution » car il importe de plus en plus des véhicules de moins bonne qualité et d'un âge avancé. Or ces véhicules sont considérés très pollueurs dans les pays développés (Matcheubou et al., 2009).

\section{* PIB industriel (IND $\left.{ }_{t}\right)$}

D'après nos estimations, le secteur industriel a un impact positif et significatif sur les émissions de $\mathrm{CO}_{2}$ par habitant au Cameroun. En effet, toute augmentation unitaire de la valeur ajoutée sectorielle rapportée au PIB est couplée à une hausse des émissions polluantes de $0,024655 \%$, ce qui est conforme à la prédiction théorique.

\section{* Densité de la population (DPOP $)$}

La variable densité de la population a un impact négatif et significatif sur les émissions de $\mathrm{CO}_{2}$ et indique que la forte concentration des populations sur un espace donné aurait tendance à réduire les émissions polluantes. En effet, l'augmentation de la densité de la population de 1 point a pour conséquence une réduction des émissions de $\mathrm{CO}_{2}$ par tête de 0,021707 points. Ce résultat pour le moins intriguant, est conforme à celui de Sharma (2010), mais contraste avec celui de Cissokho (2004).

* Consommation de carburant fossile en \% du total $\left(\mathrm{CCARB}_{\mathrm{t}}\right)$

Le signe de cette variable est positif et conforme aux prévisions. Elle indique une augmentation des émissions de $\mathrm{CO}_{2}$ de $0,013620 \%$ suite à une 
augmentation de la consommation de carburant fossile de $1 \%$. Ce résultat conforte ceux de Halicioglu (2008), Sharma (2010) et Odhiambo (2011).

\subsubsection{Relation de court terme}

\subsubsection{Résultats des tests de validation}

Le tableau suivant donne un aperçu des principaux résultats.

Tableau 9 : Récapitulatif des résultats des tests de validation du MCE

\begin{tabular}{|c|c|c|}
\hline Test & Observations & Conclusion \\
\hline $\begin{array}{c}\text { Test de détection de } \\
\text { l'autocorélation }\end{array}$ & $\begin{array}{c}\chi^{2}=2,165769 \\
\text { P-value }=0,336137>1 \%\end{array}$ & $\begin{array}{c}\text { Les erreurs sont non } \\
\text { autocorélés à } 1 \%\end{array}$ \\
\hline $\begin{array}{c}\text { Test de détection de } \\
\text { l'hétéroscédasticité }\end{array}$ & $\begin{array}{c}\chi^{2}=36,14463 \\
\text { P-value }=0,368684>1 \%\end{array}$ & $\begin{array}{c}\text { Les erreurs sont } \\
\text { homoscédastiques à } 1 \%\end{array}$ \\
\hline $\begin{array}{c}\text { Test de stabilité des } \\
\text { paramètres }\end{array}$ & $\begin{array}{c}\text { Toutes les lignes bleues évoluent à } \\
\text { l'intérieur du corridor }\end{array}$ & $\begin{array}{c}\text { Les paramètres sont tous } \\
\text { stables à 5\% }\end{array}$ \\
\hline
\end{tabular}

Source : Auteur, à partir d'Eviews 5.0

Il ressort du tableau précédent que les résidus du modèle sont non autocorrélés et homoscédastiques. De plus, les tests de stabilité des paramètres confirment que les coefficients estimés sont stables. Par conséquent, la méthode des MCO peut être appliquée à l'estimation de la relation de court terme.

\subsubsection{Estimation du MCE et interprétation des résultats}

Dans l'ensemble, les résultats du MCE sont globalement significatifs, et le coefficient de détermination dans ce cas est plus grand que celui du long terme (cf. tableau 10). Le MCE estimé est globalement significatif au seuil de $1 \%$ (Prob.(F-statistique) $=0,00014<0,001$ ) et indiquent que $70,6 \%$ des émissions de $\mathrm{CO}_{2}$ à court terme sont expliquées par les variables introduites dans le modèle. De plus, la force de rappel négative et significative au seuil de $1 \%$, est conforme aux attentes et indique qu'il existe une relation de court terme entre les émissions de $\mathrm{CO}_{2}$ par tête et la croissance économique.

Tableau 10 : Résultats de l'estimation de l'ARDL (2, 0, 1, 2, 2, 2, 1)

\begin{tabular}{|c|r|r|r|}
\hline \multicolumn{4}{|c|}{ Variable dépendante : $\mathrm{D}(\mathrm{CO})$} \\
\hline Variables indépendantes & \multicolumn{1}{|c|}{ Retard } & \multicolumn{1}{c|}{ Coefficient } & \multicolumn{1}{c|}{ Ecart type } \\
\hline $\mathrm{C}$ & & 0,162946 & 0,129052 \\
\hline \multirow{2}{*}{$\mathrm{D}(\mathrm{CO})$} & 1 & $0,493711^{* * *}$ & 0,240590 \\
\cline { 2 - 4 } & 2 & 0,211491 & 0,167757 \\
\hline \multirow{2}{*}{$\mathrm{D}(\mathrm{PIB})$} & 0 & $-0,003158$ & 0,003138 \\
\hline \multirow{2}{*}{$\mathrm{D}\left(\mathrm{PIB}^{2}\right)$} & 0 & $3,24 \mathrm{E}-06$ & $2,41 \mathrm{E}-06$ \\
\cline { 2 - 4 } & 1 & $-5,56 \mathrm{E}-08$ & $3,66 \mathrm{E}-07$ \\
\hline
\end{tabular}




\begin{tabular}{|c|c|c|c|}
\hline \multirow{3}{*}{ D(IND) } & 0 & 0,010442 & 0,007581 \\
\hline & 1 & $-0,022563 * *$ & 0,00955 \\
\hline & 2 & $-0,004022$ & 0,007644 \\
\hline \multirow{3}{*}{$\mathrm{D}(\mathrm{OUV})$} & 0 & $0,007809 * *$ & 0,002809 \\
\hline & 1 & $-0,009620 *$ & 0,003546 \\
\hline & 2 & $-0,010249^{*}$ & 0,003546 \\
\hline \multirow{3}{*}{ D(DPOP) } & 0 & $-2,648880$ & 4,31966 \\
\hline & 1 & $-0,282433$ & 8,800086 \\
\hline & 2 & 2,790322 & 4,711120 \\
\hline \multirow{2}{*}{$\mathrm{D}(\mathrm{CCARB})$} & 0 & $0,022388 * *$ & 0,007330 \\
\hline & 1 & $-0,00322$ & 0,007502 \\
\hline ECT(-1) & & $-1,773741 *$ & 0,324635 \\
\hline \multicolumn{4}{|c|}{$\mathrm{R}^{2}=0,844882$} \\
\hline \multicolumn{2}{|c|}{$\mathrm{R}^{2}$-ajusté $=0,706094$} & \multicolumn{2}{|c|}{ Prob $(F-$ stat $)=0,000148$} \\
\hline
\end{tabular}

Source : Auteur, à partir d'Eviews 5.0

Les résultats du tableau 10 indiquent qu'à court terme, les émissions de $\mathrm{CO}_{2}$ par tête à un moment précis sont déterminées par les variations à niveau et retardées des variables explicatives et expliquée. Ainsi, il ressort que les émissions par habitant à un moment donné sont influencées par le niveau d'émissions des deux périodes précédentes, dont les effets sont tous positifs. Toutefois, seules les émissions de la période précédente ont un effet positif et significatif sur les émissions actuelles.

En ce qui concerne les variables de la croissance économiques, nous pouvons noter que la variation présente des émissions de $\mathrm{CO}_{2}$ est expliquée par la variation du PIB moyen présent (qui a un impact négatif) et les variations du PIB moyen carré présent (impact positif) et décalé d'une période (impact négatif). Ce résultat tend à décrire une relation en $\mathbf{U}$ à court terme entre croissance économique et émissions de $\mathrm{CO}_{2}$, toutefois, aucun coefficient n'étant significatif, cette relation ne saurait être confirmée.

Par ailleurs, la variation actuelle du PIB industriel a un effet positif et non significatif sur les variations actuelles des émissions de $\mathrm{CO}_{2}$, qui seraient beaucoup plus expliquées par les effets négatifs des variations du PIB industriel de l'année précédente (impact significatif) et de l'année d'avant (impact non significatif). Ce résultat indique que les effets nocifs de la pollution industrielle sur la qualité de l'environnement ont tendance à diminuer au fil du temps. 
Les variations actuelles du degré d'ouverture commerciale ont un impact positif et significatif sur les variations présentes des émissions de $\mathrm{CO}_{2}$. Ceci serait dû à la forte contribution des industries extractives (hydrocarbures et bois) au solde commercial du Cameroun. Toutefois, l'impact des variations du degré d'ouverture des deux périodes précédentes serait négatif et significatif. L'effet négatif pourrait s'expliquer par la structure des biens importés.

Les effets de la variation de la densité de la population sont conforment aux résultats du long terme, sauf le deuxième retard, dont les variations ont un impact positif sur les émissions de $\mathrm{CO}_{2}$ par habitant. Toutefois, ces résultats non significatifs empêchent toute interprétation.

Enfin, la variation présente de la consommation du carburant fossile a un impact positif et significatif sur la variation des émissions de $\mathrm{CO}_{2}$ à court terme. Cet effet positif se justifie, comme pour le long terme par le développement d'un parc automobile constitué de plus en plus de véhicules très âgés et énergivores. Aussi, les variations de la consommation de carburant fossile de la période précédente ont un impact sur la variation présente des émissions de $\mathrm{CO}_{2}$ par tête. Cependant, cet effet n'est pas significatif.

\section{Conclusion}

L'objectif de ce papier était de déterminer les effets de la croissance économique et l'ouverture commerciale sur la qualité de l'environnement au Cameroun. Pour atteindre cet objectif, la modélisation adoptée est inspirée des travaux empiriques sur l'hypothèse de la Courbe Environnementale de Kuznets (CEK). L'approche méthodologique a pris appui sur la technique de cointégration par les retards échelonnés. Les résultats montrent que : la croissance économique détériore l'environnement à court terme, mais l'améliore à long terme tandis que l'ouverture commerciale détériore l'environnement. En dépit de la vérification de la CEK, le secteur industriel et la consommation de carburant fossile dégradent l'environnement au Cameroun. Ces effets, bien que marginaux sur la période d'analyse, pourraient s'amplifier au cours du processus d'industrialisation tel que prévu par le DSCE. Afin de rendre cette croissance future soutenable, les autorités gouvernementales doivent systématiser l'éducation environnementale, stimuler les investissements dans les nouvelles technologies (propres), maîtriser la consommation d'énergie fossile en réduisant la consommation du bois énergie et de carburant fossile. 


\section{References:}

1. Akpan, F. A. et Chuku, C. A. (2011): «Economic Growth and Environmental Degradation in Nigeria: Beyond the Environmental Kuznets Curve », MPRA, 27 April, pp. 10-20.

2. Banque mondiale (1992) : «Le développement et l'environnement, rapport sur le développement dans le monde ». Oxford University Press.

3. Bourbonnais, R. (2002) : «Econométrie. Manuel et Exercices corrigés », $4^{\mathrm{e}}$ éd. Dunod, Paris.

4. Chanel, A. (1993) : «De l'émergence de la question environnementale aux recherches en économie de l'environnement », DEES, n ${ }^{\circ}$ 94, décembre, pp. 5-16.

5. Chauvat, G. (2003) : «Probabilités et statistiques: exercices et problèmes corrigés », $4^{e}$ éd., Armand Colin, Paris.

6. Cissokho, L. (2004) : «Impact de la croissance économique sur la qualité de l'environnement: le cas du Sénégal», Mémoire de Diplôme d'Etudes Approfondies (D.E.A), F.A.S.E.G, Université Cheikh Anta Diop de Dakar, Sénégal.

7. Grossman, G. M. et Krueger, A. B. (1991) : «Environmental Impacts of a North American Free Trade Agreement », in P. Garder (ed.), The U.S.-Mexico Free Trade Agreement, MIT Press, Cambridge, Massachusetts, États-Unis.

8. Halicioglu, F. (2008) : «An econometric study of $\mathrm{CO}_{2}$ emissions, energy consumption, income and foreign trade in Turkey », Yedipete University, MPRA.

9. He, J. (2005) : « Impacts environnementaux de l'industrialisation et commerce international en Chine: cas de l'émission industrielle de $\mathrm{SO}_{2}$ », Thèse pour le doctorat ès sciences économique, Université d'Auvergne Clermont 1, décembre 2005.

10. Holtz-Eakin , D. and Selden T.M. (1995) : «Stoking the Fires? CO2 Emissions and Economic Growth ». Journal of Public Economics, 57, 85-101.

11. Institut National de la Statistique, (2011) : «le commerce extérieur au premier semestre $2011 »$.

12. Kuznets, S. (1955) : « Economic Growth and Income Equality », American Economic Review, vol. 45 (1), 1-28.

13. Lean, H. H. et Smyth, R. (2009) : " $\mathrm{CO}_{2}$ emissions, electricity consumption and output in ASEAN ». JEL. Development Research Unit, Discussion Paper DEVDP 09-13, Monash University.

14. Lélé, C. (2010) : «Effets des investissements dans les infrastructures sur les branches d'activités porteuses de l'économie camerounaise : 
une analyse en terme de causalité », Mémoire professionnel, Institut sous régional de la Statistique et d'Economie Appliquée (ISSEA).

15. Malthus R. (1798) : « An essay on the principle of population », Cambridge Press.

16. Matchebou, A., Yamba J. et Tatietse T. T. (2009) : «Impact du parc automobile sur la congestion du trafic et la pollution de l'air dans la ville de Yaoundé », Colloque international Environnement et Transports dans des contextes différents, Ghadaïa, Algérie, 16-18 fév. 2009. Actes ENP ed., Alger, p. 245-252

17. Meadows, D. H., D. L. Meadow, J. Randers, et W. Behrens (1972) : « The Limit to Growth ». Universe Books, New York.

18. MINEF, (2005) : «Communication initiale du Cameroun sur le climat », MINEF, Yaoundé, Cameroun.

19. Odhiambo, N. M. (2011) : «Economic Growth and Carbone Emissions in South Africa: An Empirical Investigation », International Business \& Economic Research Journal, July 2011, Volume 10, Number 7.

20. OCDE (2011): «Mondialisation, transport et environnement . Éditions OCDE. http://dx.doi.org/10.1787/9789264072930-fr.

21. OMC (1998) : «Commerce et environnement», Dossiers spéciaux, pp. 53-67

22. Pesaran, H. M., Shin, Y. and Smith R. (2001) : «Bounds testing approachesto the analysis of level of level relationships », Journal of Applied Econometrics 16: 289-326 (2001).

23. Phu Nguyen V. et Azomahou T., (2002) : «déforestation, croissance économique et population : une étude sur données de panel », BETAThème, université Louis Pasteur, JEL : C23 ; O13 ; Q23 ; R15.

24. Saboori B. and Soleymani A., (2011) : « $\mathrm{CO}_{2}$ emissions, economic growth and energy consumption in Iran: A cointegration approach». International Journal of Environmental Sciences, volume 2, $\mathrm{N}^{\circ} 1$, 2011

25. Shafik, N. and Bandyopadhyay, S. (1992) : « Economic growth and environmental quality: times series and cross-country evidence », Bachground paper for the World Development Report 1992 (Washington, DC: The World Bank, 1992).

26. Sharma, S. S. (2010) : «Determinants of carbon dioxide emissions : Empirical evidence from 69 countries », Applied Energy 88(2011) 376-382

27. Stern N. (2007) : «The Economics of Climate Change: The Stern Review », United Kingdom: Cambridge University Press, 2007. 
Annexe 1 : Résultat de la détermination du retard optimal de Pesaran

\begin{tabular}{|c|c|c|c|c|c|c|}
\hline \multicolumn{7}{|c|}{$\begin{array}{l}\text { VAR Lag Order Selection Criteria } \\
\text { Endogenous variables: CO2 PIB PIB } \\
\text { Exogenous variables: C } \\
\text { Date: 08/23/13 Time: } 03: 00 \\
\text { Sample: } 19712011 \\
\text { Included observations: } 37\end{array}$} \\
\hline Lag & LogL & LR & FPE & AlC & Sc & $\mathrm{HQ}$ \\
\hline $\begin{array}{l}0 \\
1 \\
2 \\
3\end{array}$ & $\begin{array}{l}-1034.417 \\
-657.1026 \\
-564.0523 \\
-437.9441\end{array}$ & $\begin{array}{c}\text { NA } \\
591.4658 \\
110.6544 \\
102.2499^{*}\end{array}$ & $\begin{array}{l}6.61 e+15 \\
1.36 e+08 \\
16946683 \\
656005.3^{*}\end{array}$ & $\begin{array}{l}56.29281 \\
38.54609 \\
36.16499 \\
31.99698^{*}\end{array}$ & $\begin{array}{l}56.59758 \\
40.98423 \\
40.73651 \\
38.70188^{\star}\end{array}$ & $\begin{array}{l}56.40026 \\
39.40565 \\
37.77667 \\
34.36077^{*}\end{array}$ \\
\hline \multicolumn{7}{|c|}{$\begin{array}{l}\text { * indicates lag order selected by the criterion } \\
\text { LR: sequential modified LR test statistic (each test at } 5 \% \text { level) } \\
\text { FPE: Final prediction error } \\
\text { AIC: Akaike information criterion } \\
\text { SC: Schwarz information criterion } \\
\text { HQ: Hannan-Quinn information criterion }\end{array}$} \\
\hline
\end{tabular}

Annexe 2 : Spécification du Modèle à Correction d'Erreur de Pesaran et al. (2001)

$$
\begin{aligned}
& \Delta C O_{t}=\alpha_{o}+\sum_{i=1}^{2} \alpha_{1} \Delta C O_{t-i}+\alpha_{2} \Delta P I B_{t}+\sum_{i=0}^{l} \alpha_{3 i} \Delta P I B_{t-1}^{2}-i+\sum_{i=0}^{2} \alpha_{4 i} \Delta I N D_{t-i}+\sum_{i=0}^{2} \alpha_{5 i} \Delta O U V_{t-i} \\
& +\sum_{i=0}^{2} \alpha_{6 i} \Delta D P O P_{t-i}+\sum_{i=0}^{l} \alpha_{7 i} \Delta C C A R B_{t-i}+\pi E C T_{t-1}+\varepsilon t
\end{aligned}
$$

$\underline{\text { Annexe } 3}$ : Résultats des tests CUSUM et CUSUMQ du modèle de long

terme

\section{Test CUSUM}

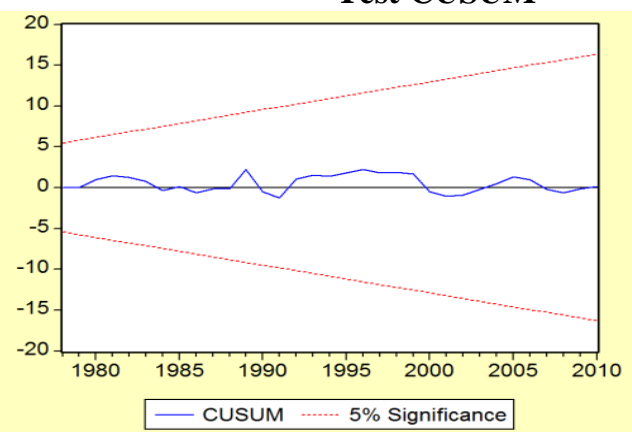

Test CUSUMQ

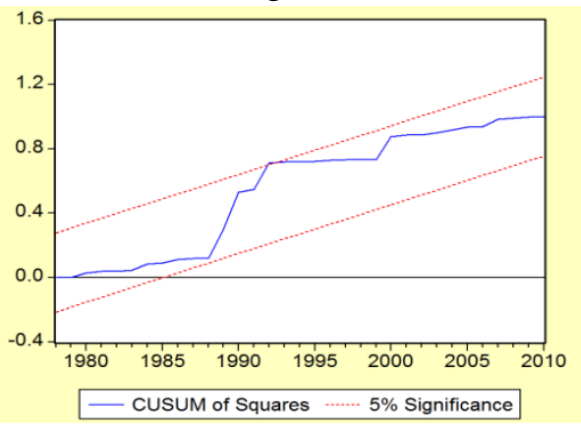

$\underline{\text { Annexe } 4}$ : Résultats des tests de validation du Modèle à Correction d'Erreur

1. Test de détection de l'autocorrélation Breusch-Godfrey Serial Correlation LM Test:

\begin{tabular}{cccc}
\hline \hline F-statistic & 0.528476 & Probability & 0.598878 \\
Obs*R-squared & 2.165769 & Probability & 0.338617 \\
\hline \hline
\end{tabular}




\section{Test de détection de l'hétéroscédasticité}

White Heteroskedasticity Test:

\begin{tabular}{ccll}
\hline \hline F-statistic & 2.485663 & Probability & 0.328083 \\
Obs*R-squared & 36.14463 & Probability & 0.368684 \\
\hline \hline
\end{tabular}

3. Tests CUSUM et CUSUMQ

Test CUSM

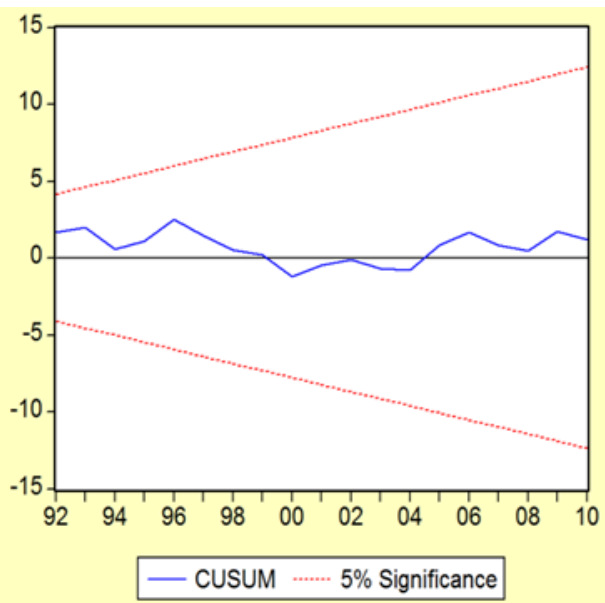

Test CUSUMQ

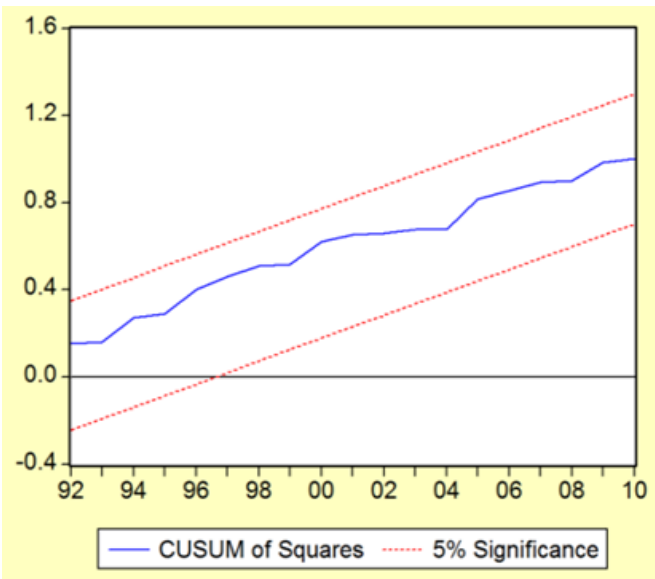

